\title{
Cáncer de Seno en la Clínica San Pedro Claver de Bogotá, 2004
}

\author{
Mario A. González-Mariño \\ Médico. Esp. Clínica San Pedro Claver. Facultad de Medicina, Fundación Universitaria San Martín, Bo- \\ gotá, Colombia. E-mail: marioar90@hotmail.com
}

Recibido 23 Febrero 2006/Enviado para Modificación 2 Junio 2006/Aprobado 25 Junio 2006

\section{RESUMEN}

Objetivo Revisar los casos nuevos de cáncer de seno atendidos durante el año 2004 en la Clínica San Pedro Claver, Bogotá, Colombia.

Material y Métodos La captación de las pacientes se hizo a través de la junta de seno, actividad a la cual asisten todas las pacientes nuevas de este servicio. Para garantizar la inclusión de la totalidad de ellas, además se realizó búsqueda activa de los casos entre las pacientes hospitalizadas, consulta externa, registros de programación de cirugía y reportes de patología. Los datos fueron registrados en un archivo Excel y evaluados mediante el programa EPIINFO.

Resultados Se encontraron 220 casos de cáncer de mama. La edad promedio fue 59 años, el $18 \%$ tenía menos de 50 años. El carcinoma ductal infiltrante con el $84,1 \%$ de los casos fue el tipo histológico más frecuente. La distribución según estadíos concentró el mayor número de pacientes en los estados 0 al II en el 62,7\%. Los tumores midieron más de 2 centímetros en el $78 \%$ de ellos. El tratamiento quirúrgico se realizó en el $83 \%$ de las pacientes y el de quimioterapia en el $60 \%$. Sin embargo, en las mujeres mayores de 69 años tanto la cirugía (58,3 \%) como la quimioterapia $(13,9 \%)$ fueron indicados en proporciones inferiores a las de las mujeres con menor edad $(p<0.05)$. En el 40,9\% de los casos, se indicó hormonoterapia.

Conclusiones El registro institucional de Cáncer de la Clínica San Pedro Claver permite conocer las características personales, clínicas y modalidades terapéuticas pertenecientes a los casos nuevos de cáncer de mama atendidas durante el año 2004 en esta institución. La mayoría de los casos corresponden a estados tempranos. Sin embargo, se debe continuar en el empeño de detectar un mayor número de lesiones no palpables. Se hace necesario evaluar los tratamientos indicados en las mujeres mayores de 69 años con respecto a lo encontrado en los grupos más jóvenes y la calidad del procedimiento, laboratorio, reporte de los receptores hormonales y la decisión terapéutica que puedan explicar la baja proporción del tratamiento con hormonoterapia. 
Palabras Clave: Cáncer de mama, registros, registros de hospitales, registros médicos (fuente: MeSH, BIREME).

\section{ABSTRACT}

Breast cancer in the Pedro Claver hospital in Bogotá, 2004

Objective Reviewing new cases of breast cancer attending Clinica San Pedro Claver, Bogotá, Colombia, during 2004.

Material and Methods Patients were recruited from the meeting offered by the service which all new breast cancer patients attend. Hospitalised patients, outpatients, clinical meetings, consulting rooms, operating room schedules and pathology reports were consulted to ensure that a true crosssection was included. The information was recorded in an Excel file and analysed using EPIINFO software.

Results Breast cancer was diagnosed in 220 patients. Average age was 59; $18 \%$ were aged les than 40 . Infiltrative ductal cancer $(84,1 \%)$ was the most frequently found histological type. Distribution by stage showed that most patients presented stage 0 to stage II $(62,7 \%)$. Tumours measured 2 or more centimetres in $78 \%$ of the patients. $83 \%$ of the patients had surgery and $60 \%$ chemotherapy. However, these scores were lower in patients aged more than 69 (58,3 \% for surgery and just 13,9\% for chemotherapy, $\mathrm{p}<0.05)$. Hormonotherapy was prescribed for $40,9 \%$ of the patients.

Conclutions Breast cancer is the more frequent found carcinoma. Clinica San Pedro Claver's cancer records revealed the personal, clinical and therapeutic characteristics of new female breast cancer patients attending this hospital during 2004. Most patients were in early breast cancer stages; however, detecting a greater number of non-palpable lesions should still be stressed. Treating elderly patients (those older than 69 regarding that found for younger patients) and the quality of procedures, hormonal receptor reports and therapeutic decision should also be evaluated in an attempt to explain the low percentage of hormone treatment currently being used in the hospital.

Key Words: Breast cancer, hospital records, data collection, medical records (source: MeSH, NLM).

L

a Clínica San Pedro Claver de Bogotá, Colombia, pertenece al Instituto de Seguros Sociales. El registro institucional de esta institución reúne datos concernientes a los pacientes de esta entidad que presentan patología neoplásica. En el año 2004, se registraron 725 pacientes que presentaron alguna de las patologías oncológicas en evaluación (cánceres ginecológicos, mama, gástrico y vías urinarias) En este informe se presentan los pacientes con diagnóstico de cáncer de mama. 


\section{MÉTODOS}

Se realizó un estudio descriptivo de las características personales y de tratamiento en pacientes con diagnóstico de primera vez de cáncer de seno que asistieron a la Clínica San Pedro Claver, en el año 2004.

La captación de las pacientes se hizo a través de la junta del servicio de seno, actividad a la cual asisten todas las pacientes nuevas de este servicio. Para garantizar la inclusión de la totalidad de ellas, además se realizó búsqueda activa de los casos en las pacientes hospitalizadas, registros de programación de cirugía, reportes de patología y en consulta externa, cumpliendo con el criterio de exhaustividad (captura de todos los casos nuevos).

La codificación de morbilidad se hizo de acuerdo con la Clasificación Internacional de Enfermedades y problemas relacionados con la salud (CIE10) y para codificar el tipo morfológico o histológico del tumor según el diagnóstico anatomo-patológico se siguió la Clasificación Internacional de Enfermedades para oncología (CIE-0) $(1,2)$

Los datos fueron registrados en un archivo Excel y evaluados mediante el programa EPIINFO versión 2002. Se emplean medidas estadísticas de posición (porcentaje), tendencia central (promedio aritmético) y de variabilidad (rango y desviación estándar).

\section{RESULTADOS}

EL registro de cáncer de 2004 de la Clínica San Pedro Claver posee datos de 220 pacientes con cáncer de mama, cuyo diagnóstico se realizó por estudio histológico del tumor primario. Del total de pacientes, 8 fallecieron en el mismo año.

La mayoría de la mujeres evaluadas residía en Bogotá principalmente en las zonas de Engativá (13,6\%), Kennedy (13,6\%), Suba (10\%, y San Cristobal $(7,7 \%)$. La distribución por edades se presenta en la Figura 1.

En 67 pacientes (30,5\%) se encontró el estado clínico IIb y en 32 mujeres $(14,5 \%)$ el estado IIa. La distribución por estado se encuentra en la Figura 2.

El carcinoma ductal infiltrante fue el tipo histológico predominante, con el $87,7 \%$ del total, como se indica en la Tabla 1 . El termino "SAI" significa "sin otra especificación"; el cual es usado por la CIE-0 para el término to- 
pográfico o morfológico que no este siendo modificado por ningún adjetivo o cuando va acompañado de un adjetivo no descrito en la clasificación.

La distribución según el grado histológico se muestra en la Figura 3.

Tabla 1. Distribución del cáncer de seno según tipo histológico

\begin{tabular}{lcc}
\hline \multicolumn{1}{c}{ Tipo Histológico } & $\begin{array}{c}\text { Número de } \\
\text { casos }\end{array}$ & $\%$ \\
\hline Carcinoma ductal infiltrante & 193 & 87,7 \\
Carcinoma ductal in situ & 13 & 5,9 \\
Carcinoma lobulillar infiltrante & 3 & 1,4 \\
Adenocarcinoma mucinoso & 3 & 1,4 \\
Carcinoma papilar, SAI & 2 & 0,9 \\
Otros & 6 & 2,7 \\
\hline \multicolumn{1}{c}{ TOTAL } & 220 & 100,0 \\
\hline
\end{tabular}

Los tumores se ubicaron en el cuadrante superior externo en 94 casos $(42,7 \%)$, lesiones solapadas que comparten los límites de dos o más subcategorías de la mama en 60 tumores $(27,3 \%)$ y en el cuadrante superior interno en 28 tumores $(12,7 \%)$. Las demás localizaciones tumorales tuvieron una baja frecuencia. Estos tumores se ubicaron en idéntica proporción en los dos senos.

CANCER DE SENO EN LA CLINICA SAN PEDRO CLAVER, 2004

Figura 1. Casos según edad

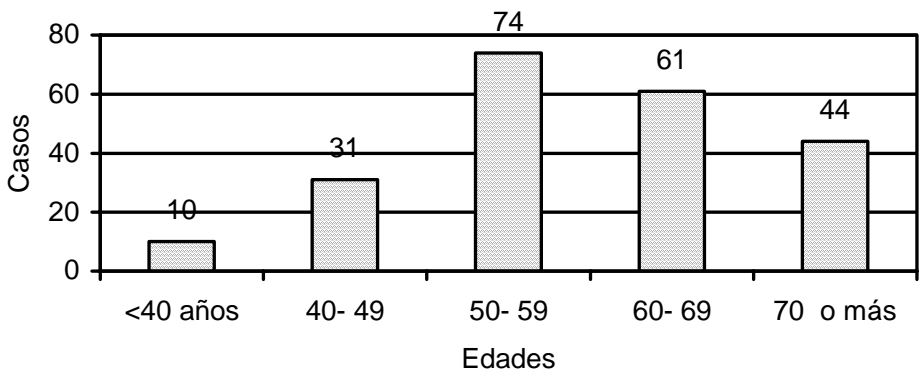

El tamaño de los tumores se distribuye como muestra la Figura 4. Las pacientes presentaban metástasis en ganglios axilares homolaterales móviles, N1, en 113 casos $(51,4 \%)$ y no presentaban metástasis en ganglios linfáticos regionales, N0, en 77 (35\%). En 11 pacientes $(5 \%)$ se encontró metástasis a ganglios axilares homolaterales estando fijos entre sí o a otras es- 
tructuras, $\mathrm{N} 2$, y en tres pacientes $(1,4 \%)$ había compromiso de ganglios supraclaviculares ipsilaterales. En los casos restantes, no se encontró información.

Figura 2. Casos según estados del cáncer

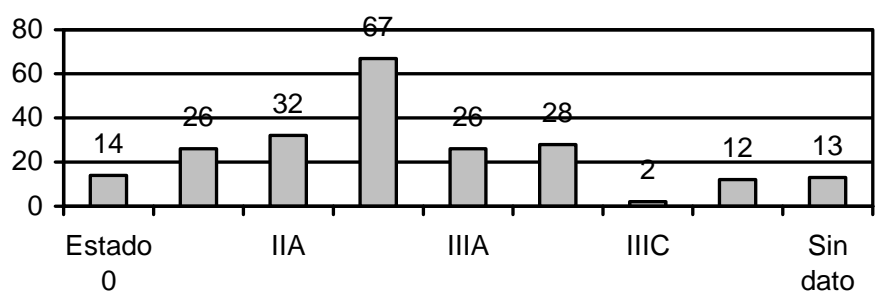

Figura 3. Casos según grado de diferenciación

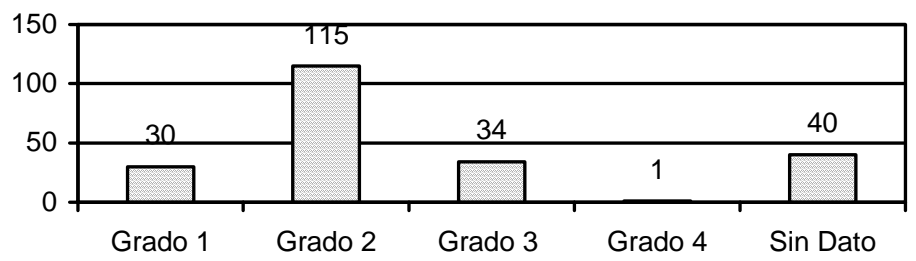

Figura 4. Casos según características del tumor

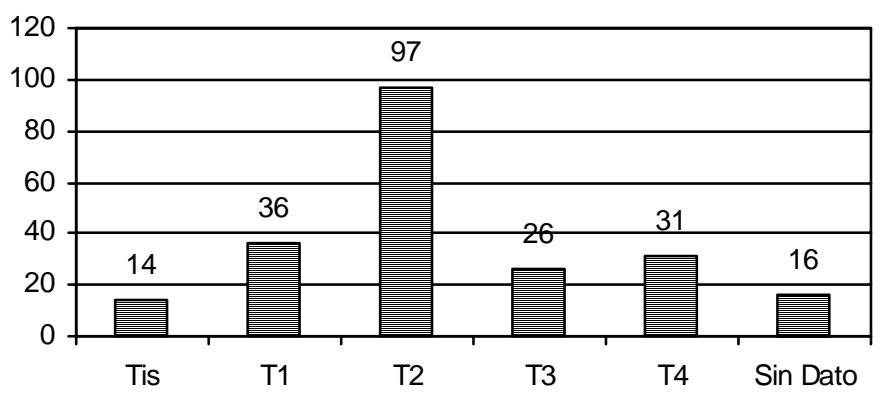

Metástasis a distancia

En 12 de las 220 pacientes, hubo metástasis a distancia. Los sitios con compromiso metastásico fueron hueso $(2,7 \%)$, hígado $(1,4 \%)$, pulmón $(0,9$ $\%)$ y encéfalo $(0,45 \%)$.

\section{Tratamiento}

El tratamiento más frecuente fue la cirugía realizada en 184 pacientes $(83,6$ $\%$, quimioterapia en 132 pacientes $(60 \%)$, radioterapia en $84(38,2 \%)$ y 
hormonoterapia en 90 pacientes $(40,9 \%)$. Además de estos tratamientos, 208 pacientes $(94,5 \%)$ recibieron algún otro tratamiento como psicología, rehabilitación, cirugía plástica o psiquiatría.

La indicación de tratamiento con cirugía $(58,3 \%)$ o quimioterapia $(13,9$ \%) fue menor en las mujeres mayores de 69 años que en las más jóvenes, mostrando diferencias estadísticamente significativas $\mathrm{p}<0.05$, las cuales no se encontraron en la distribución por estados.

\section{DISCUSION}

Este estudio describe pacientes afiliadas al sistema de salud en la modalidad del régimen contributivo que asistieron a la Clínica San Pedro Claver para el manejo de cáncer de mama. En esta institución, esta patología fue la más frecuente entre los cánceres en al año 2004 con 220 casos superando al cáncer de cérvix que se encontró en 115 mujeres.

La distribución por edad muestra que el 18,6 \% (41 mujeres) de estas pacientes son menores de 50 años, lo cual es importante al evaluar programas de prevención secundaria de esta enfermedad, en la proyección del pronóstico y en el enfoque terapéutico de este grupo de mujeres

Los estado 0 hasta el II son los predominantes con el 58,6 \%. Para el caso del estado 0 las lesiones fueron no palpables diagnosticadas mediante biopsia por arpón en 7 de los 9 casos. El hallazgo en estados tempranos representa un cambio dentro de la misma institución que en un estudio entre 1982 y 1993 había reportado una mayoría de pacientes en los estados III y IV(55 \%) (3) y se acercan más a lo reportado por servicios de patología mamaria con alto índice de biopsia de lesiones no palpables como lo publicado por Lifrange sobre su servicio en Lieja, Bélgica, donde constituyen el $30 \%$ de las biopsias y en el que más del $20 \%$ de los cánceres operados tienen un tamaño menor de $1 \mathrm{~cm}$ (Estado I)(4,5).

En este registro, en las mujeres mayores de 69 años la cirugía y la quimioterapia no tuvieron la misma frecuencia de indicación que en las edades menores, sin que esto fuera explicable para el caso de la cirugía por cambios en la distribución de pacientes en estado IV a quienes por su pronóstico se les excluye usualmente de este tratamiento. Tampoco para el caso de la quimioterapia estuvo asociada con la distribución de los estados 0 en el cual no está indicada o en el estado I en el que no se recomienda en caso de pacientes de riesgo mínimo $(6,7)$ Lo anterior coincide con otros reportes que señalan la misma tendencia en cuanto a la disminución en el tratamiento para 
este grupo de edad $(8,9)$.Sin embargo, el registro no evalúa la existencia de patologías concomitantes que en algunas pacientes puede explicar la reticencia en la indicación de estos procedimientos.

La baja frecuencia de hormonoterapia en esta revisión $40 \%$ ( se espera que 70 a $80 \%$ de cánceres mamarios tengan receptores hormonales positivos) (10) suscita inquietud respecto al proceso que conduce al reporte de los receptores de estrógenos y progesterona, cuyo resultado falsamente negativo priva a algunas pacientes de un medicamento que puede mejorar su sobrevida $(7,11)$. Un paso adicional a evaluar a nivel institucional es la disminución en el umbral para ordenar la hormonoterapia (actualmente en el $10 \%$ de células positivas)

\section{REFERENCIAS}

1. Percy C, Van Holten V, Muir C. Clasificación internacional de enfermedades para oncología. Segunda edición. Ginebra: Organización Mundial de la Salud (OMS) ; 1990

2. Organización Panamericana de la Salud. Clasificación estadística internacional de enfermedades y problemas relacionados con la salud (CIE-10).(décima revisión).Publicación científica No 554. Washington, D.C. OPS;1995.

3. Márquez G, González MA, Abaunza H. Cáncer mamario. Clínica San Pedro Claver I.S.S. En: Cáncer mamario, Abaunza H. (editor). Colombia: Lerner Ltda.; 1997.

4. Lifrange E, Colin C. La Chirurgie mammaire supraconservatrice. J Gynecolog Obstet Biol Reprod 2000;29 :285-287.

5. González M.A. Diagnóstico de lesiones no palpables de seno. Revista Colombiana de Cirugía 2002; 17(4):224-231.

6. Mincey BA., Palmieri FM, Perez EA. Adjuvant Therapy for Breast Cancer: Recommendations for Management Based on Consensus Review and Recent Clinical Trials. The Oncologist 2002; 7:246-250.

7. Goldhirsch A, Wood WC, Gelber RD, Coates AS, Thürlimann B, Senn H. Meeting highlights: updated international expert consensus on the primary therapy of early breast cancer. J Clin Oncol 2003; 21.3357-3365.

8. Giordano SH, Hortobagyi GN, Kau SW, Theriault RL, Bondy ML. Breast cancer treatment guidelines in older women .J Clin Oncol. 2005 Feb 1;23(4):78391.

9. Kemeny MM, Bush-Devereaux E, Merriam LT, O'Hea BJ. Cancer Surgery in the elderly. Hematology/Oncology Clinics of North America 2000;14(1):169192.

10. Keen JC, Davidson NE. The biology of breast carcinoma. Cancer 2003;97(3 Suppl):825-33.

11. Hortobagyi GN. Opportunities and Challenges in the Development of Targeted therapies. Seminars in Oncology 2004;31(1 Suppl 3):21-27. 